\title{
Determinación de la resistencia a esfuerzo cortante en ensayos de flexión a paneles sándwich PUR: análisis de las dificultades y simulación por elementos finitos
}

\section{Measurement of shear strength resistance in flexion test on PUR sandwich panels: analysis of difficulties and finite element method simulation}

\author{
J. M. Chillón Moreno $^{(*)}$, J. Sánchez Montero ${ }^{(*)}$, J. Pecharromán Sacristán ${ }^{(*)}$
}

\section{RESUMEN}

La utilización de elementos constructivos compuestos, combinando distintos materiales con características complementarias, se ha extendido cada vez más. Los paneles sándwich, compuestos por capas externas de metal que les aporta resistencia y núcleo de espuma aislante térmica, que proporciona cualidades que mejoran el confort térmico en el interior de todo tipo de construcciones, se utilizan en cerramientos y cubiertas que conforman la envolvente de los edificios. De los distintos controles de calidad a que se han de someter, en este artículo se propone una mejora al indicado en las normas para la determinación de la resistencia al esfuerzo cortante, consiguiendo evitar muchos resultados anómalos obtenidos por la utilización de placas rígidas en los apoyos. Finalmente, tanto el problema observado como la solución propuesta, se modelizan y simulan mediante el método de elementos finitos.

Palabras clave: paneles sandwich; metal; aislantes; PUR; PIR; ensayo cortante.

\section{ABSTRACT}

The use of the composite construction elements combining different materials with complementary characteristics, it has spread increasingly. The sandwich panels composed by external metallic sheets that they contributes resistance and core of rigid insulating thermal foam, that provides qualities that improve the thermal comfort inside all kinds of constructions. They are in use in closings and covers that shape the surrounding one of the buildings. Of the different quality controls to which they have to surrender. In this article one proposes an improvement to the indicated one in the procedure for the determination of the resistance to the shear strength, Managing to avoid many anomalous results obtained by the utilization of rigid plates in the supports. Finally, so much the problem observed as the proposed solution, they are modeled and simulate by means of the method of finite elements.

Keywords: sandwich panels; metal; insulating; PUR; PIR; tests shear strength.

(*) Instituto de Ciencias de la Construcción Eduardo Torroja - CSIC. Madrid (España).

(**) Universidad Politécnica de Madrid (España).

Persona de contacto/Corresponding author: jmchillon@ietcc.csic.es (J. M. Chillón Moreno).

ORCID: http://orcid.org/oooo-0oo1-9105-326o (J. M. Chillón Moreno); http://orcid.org/oooo-0002-4334-0553

(J. Sánchez Montero); http://orcid.org/oooo-0002-2609-6069 (J. Pecharromán Sacristán)

Cómo citar este artículo/Citation: Chillón Moreno, J. M., Sánchez Montero. J., Pecharromán Sacristán, J. (2017). Determinación de la resistencia a esfuerzo cortante en ensayos de flexión a paneles sándwich PUR: Análisis de las dificultades y simulación por elementos finitos. Informes de la Construcción, 69(547): e208, doi: http://dx.doi.org/10.3989/id56079.

Copyright: (c) 2017 CSIC. Licencia / License: Salvo indicación contraria, todos los contenidos de la edición electrónica de Informes de la Construcción se distribuyen bajo una licencia de uso y distribución Creative Commons Attribution License (CC BY) Spain 3.o. 


\section{INTRODUCCIÓN}

La utilización de paneles tipo sándwich se ha extendido en las últimas décadas, aumentando sus aplicaciones. Por ello, los fabricantes han optado por certificar sus productos, induciendo mejoras y actualizaciones en la normativa que controla los mismos. Este tipo de paneles se puede fabricar con diversas combinaciones de materiales en las capas externas y el núcleo, pero nos vamos a referir a los más extendidos y que adoptan la denominación que da título a esta aportación. En general están compuestos por dos finas capas exteriores de metal que aportan resistencia mecánica y un núcleo de distintos espesores, en función de su uso, muy superiores a los de las chapas metálicas (Figura 1). El núcleo que ha de cumplir una función de aislante térmico es de distintos materiales aislantes susceptibles de ser conformados y confinados entre las dos chapas metálicas. Las chapas metálicas pueden ser de: aluminio, acero, cobre y acero inoxidable y pueden ser lisas, ligeramente perfiladas o nervadas. Tienen distintos tratamientos y recubrimientos y los espesores más utilizados oscilan alrededor de 0,5 o o,6 mm. Los núcleos pueden ser de: espuma de poliuretano rígido (PUR o PIR), poliestireno expandido, espuma de poliestireno extrusionado, espuma fenólica, vidrio celular o lana mineral. Los espesores en función de su uso pueden ir desde 30 a $300 \mathrm{~mm}$.

La fabricación de los paneles sándwich ha estado regulada desde sus comienzos, en 1990 la ECCS (European Convention for Constructional Steelwork) T.C.7-W.G 7.4 (1), edita su Preliminary European Recommendations for Sandwich Panels, en el que se habla del trabajo encomendado en 1983 para preparar Recomendaciones Europeas para el cálculo y aplicación de estos elementos compuestos.

A partir de la Directiva 89/106/CEE del Consejo de 21 de diciembre de 1988, relativa a la aproximación de las disposiciones legales, reglamentarias y administrativas de los Estados miembros sobre los productos de construcción (2), se tiende a la armonización técnica para ello entidades como la UEAtc (Union Européenne pour l'Agrément technique dans la construction) fueron pioneras en la armonización de especificaciones técnicas para productos innovadores de construcción.

En el caso de los paneles sándwich sus investigaciones fueron aprovechadas por entidades surgidas mucho después, como la EOTA (European Organisation for Technical Approvals) para la redacción de la Guía DITE 016 parte (3) y el CEN (Centre Européen de Normalisation) para la redacción de la Norma UNE EN 14509 (4) (5). Esta última es una norma armonizada, regula actualmente el producto y los ensayos a realizar.

Con la aparición del Reglamento de Productos de Construcción (305/2011, de 9 de marzo), cuya entrada en vigor se ha producido, de forma efectiva, a partir del 1 de julio de 2013, se ha derogado la Directiva de Productos de Construcción DPC (89/106/CEE). Por lo que a partir de este momento se han de adaptar las normas armonizadas, como la que nos ocupa, al nuevo Reglamento en sus referencias a la anterior Directiva.

Este cambio afecta al marcado CE y es necesaria una cierta adaptación al nuevo Reglamento, pero éste no es el objeto actual del presente trabajo.

La caracterización de los paneles autoportantes se ha venido realizando según lo indicado en la Guía ETAG-O16 (European Technical Approvals Guide) (3), publicada por la Organización EOTA, y de las Normas 41950:1994 (hoy ya obsoleta) (4) y la UNE-EN 14509:2014 Paneles sándwich aislantes autoportantes de doble cara metálica. Productos hechos en fábrica. Especificaciones, realizada por el CEN/TTC 128 y que sustituye a la anterior (5).

A pesar de que la literatura no es muy extensa, algunos autores han estudiado el comportamiento mecánico de los paneles sándwich, en algunos casos han apuntado el problema

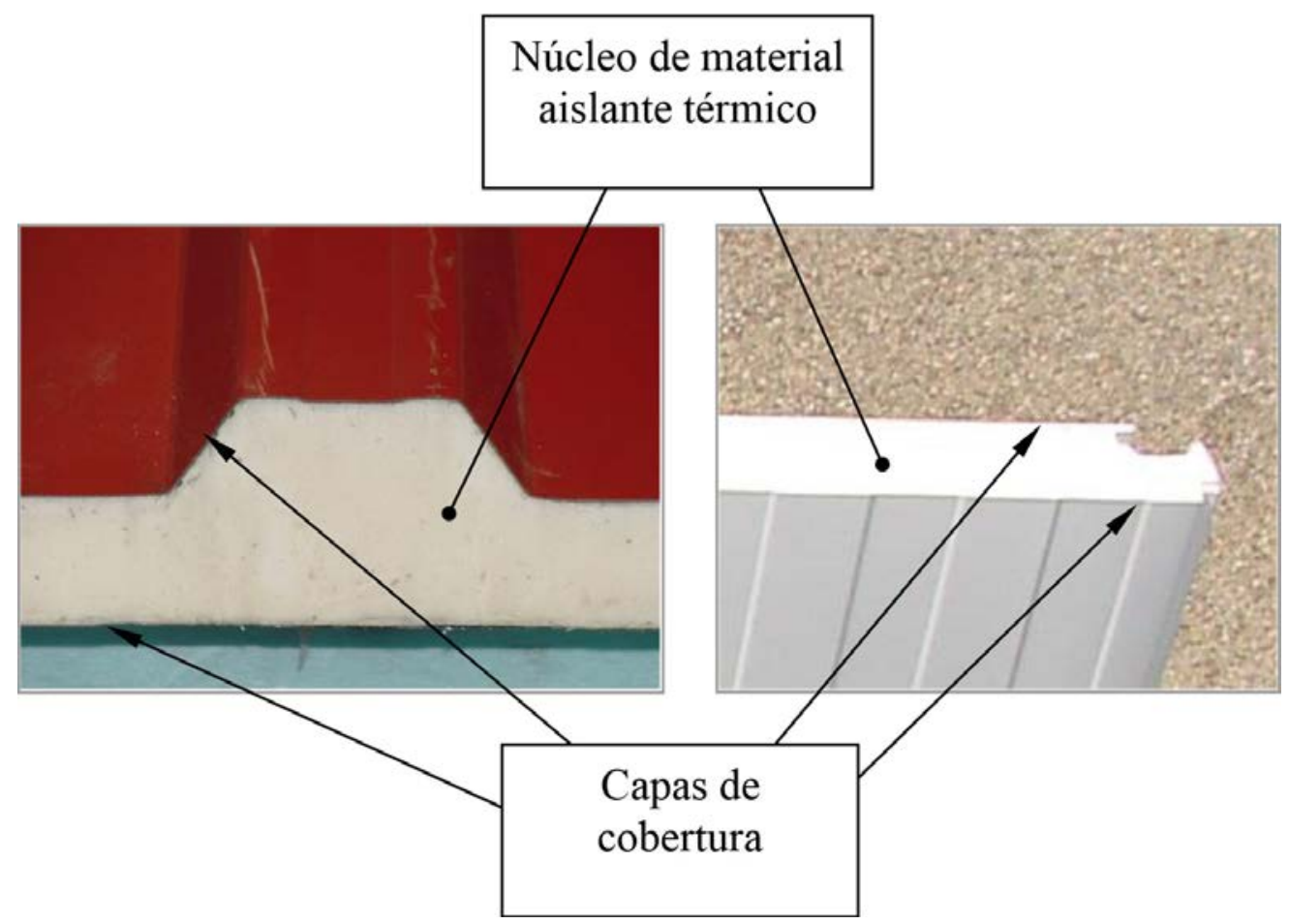

Figura 1. Paneles: nervado y plano perfilado. 
con los apoyos aunque no profundizan en sus causas como, por ejemplo, autores estudiosos como Oliver Raabe (6) y P. Hassinen (7). Otros autores abordan el problema que surge en los apoyos desde el punto de vista teórico, pero sin abordar exactamente la solución a este problema como, por ejemplo, el eminente investigador Klaus Berner (8) referencia en la investigación del comportamiento de este tipo de paneles. Otros autores, como los indicados en las referencias (9) (10) (11) (12) (13) (14) (15) (16) (17) (18) (19) (20) (21), aportan sus estudios análogos aplicados a paneles celulares o con núcleo en nido de abeja, más extendidos en países como Estados Unidos.

De la experiencia de los múltiples ensayos realizados en el Laboratorio de Instalaciones del Instituto de Ciencias de la Construcción Eduardo Torroja se observan los problemas que surgen en la realización de cada uno de los ensayos indicados en las normas. Uno de los ensayos a realizar más significativos es el Ensayo de Resistencia a esfuerzo cortante sobre el material del núcleo, indicado en el apartado A.3 de la norma UNE-EN 14509:2014. El citado ensayo plantea unos problemas al clavarse las chapas metálicas indicadas por la norma para el reparto de las cargas, provocando el colapso de las chapas de los paneles e impidiendo alcanzar la rotura por cortante deseada.

En este artículo se indica una solución adoptada que minimiza el citado problema, así como la modelización por el método de elementos finitos tanto del problema como de la solución.

\section{METODOLOGÍA}

Para el cumplimiento del objetivo se hace necesaria la realización de ensayos adecuados que reproduzcan el comportamiento de los citados elementos instalados en su ubicación definitiva. Para ello se realizan diferentes ensayos relativos a los propios materiales y ensayos funcionales relativos a la respuesta de los paneles ya conformados.

En este caso para el objeto de este trabajo reproducimos el Ensayo de Resistencia a esfuerzo cortante sobre el material del núcleo, indicado en el apartado A.3 de la norma UNE-EN 14509:2014.

En este trabajo se tratan los paneles con núcleo de espuma rígida PUR o PIR ya que son los más utilizados y las características de ambos son muy similares (9) (10).

\subsection{Resistencia a esfuerzo cortante según UNE-EN 14509:2014 Anexo A3}

En esta norma la resistencia a la cizalladura se define como el cociente entre la carga de rotura aplicada y dos veces la sección de la probeta ensayada. El módulo de elasticidad a la cizalladura es el determinado a partir de la flexión de la probeta de ensayo medida en la zona ideal de carga, sin precisar el procedimiento de cálculo.

\subsubsection{Aparatos, equipos auxiliares y materiales}

Máquina de compresión: Se utiliza la máquina universal de ensayos de tracción-compresión.

Accesorios: Un perfil rígido para el reparto de la carga en dos puntos. Cuatro rodillos rígidos de $30 \mathrm{~mm}$ de diámetro, dos para los apoyos del perfil rígido de reparto de la carga y dos para el apoyo de la probeta. Cuatro placas rígidas de la anchura de la probeta por una longitud de $\mathrm{Ls}=60$ a $100 \mathrm{~mm}$ con un espesor de 8 a $12 \mathrm{~mm}$ para interponer entre los rodillos y los cuatro contactos con el panel. Las dimensiones de las mismas se elegirán para evitar el posible aplastamiento local. Si con cualquiera de las combinaciones de dimensiones de las placas rígidas se continúa produciendo el aplastamiento, se sustituyen por otras formadas por varias finas placas de acero templado que se adaptan a la curvatura del panel deformado y evitan así al empotramiento de los bordes de las placas rígidas.

\subsubsection{Probetas de ensayo}

Las probetas tienen el espesor del producto acabado, incluyendo sus capas de cobertura y revestimiento de las mismas: la anchura de la probeta $B=100 \mathrm{~mm} \pm 2 \mathrm{~mm}$; longitud de la probeta: $L p=1.100 \mathrm{~mm}$; el espesor de la probeta, medido entre las fibras neutras de sus capas de cobertura $e=60 \mathrm{~mm}$.

Las probetas se cortan de las muestras, en dirección longitudinal del panel, con una sierra de cinta con hoja de 32 dientes por pulgada, de forma que sus caras cumplan con unas tolerancias de paralelismo y planeidad entre ellas inferior al o,5 $\%$ del lado de la probeta, con un máximo de 0,5 mm.

El número de probetas de ensayo fue de seis. Antes del ensayo las probetas permanecieron al menos 24 horas en el ambiente del laboratorio a una temperatura de $23 \pm 2^{\circ} \mathrm{C} \mathrm{y}$

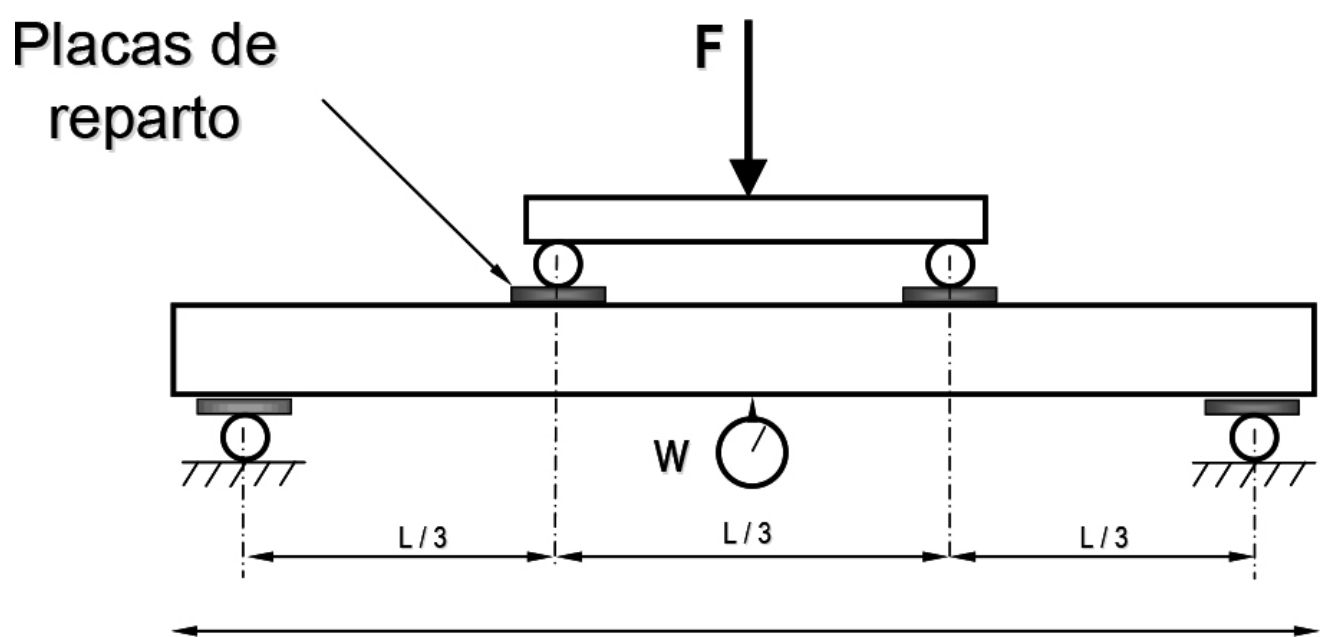

Figura 2. Ensayo a cortante. Flexión 4 puntos. 
una humedad relativa del $50 \pm 5 \%$. También se miden las dimensiones de las probetas de acuerdo con la norma UNE-EN 12085 y se registran.

Las probetas se centran entre los rodillos y apoyos de la máquina de compresión. Se apoya sobre dos rodillos rígidos e indeformables, uno de ellos horizontal fijo y el otro basculante para poder acoplarse a la probeta en el caso de que estuviera afectada de un posible alabeo y así evitar un apoyo parcial sobre una parte de la probeta.

Entre la probeta y los rodillos, tanto de apoyo como de carga, se interponen las placas indicadas en el apartado 2.1.1.

La separación entre apoyos es de una distancia $L$, en principio igual a $1.000 \mathrm{~mm}$. Esta distancia se podrá ir reduciendo, en el caso de que la rotura no se produjera por cortante, hasta conseguirlo.

Sobre la probeta, centrados sobre su eje longitudinal y separados una distancia de $1 / 3$ de $L$, se colocan las otras dos placas, sobre las que se apoya el perfil para transmitir la carga por medio de los dos rodillos.

El perfil está fijado al plato superior de la máquina de compresión y se calibra la máquina a cero de fuerza, teniendo en cuenta el peso de los posibles elementos auxiliares en el caso de que se apoyaran sobre la probeta (Figura 2).

En la zona inferior de la probeta, en el centro entre apoyos, se fija el captador de desplazamiento, en este caso del tipo denominado «de hilo».

La velocidad de avance de la máquina es del $10 \%$ del espesor $\pm 25 \% \mathrm{~mm} / \mathrm{min}$. Se registra la curva de fuerza/desplazamiento, obteniéndose la resistencia a la cizalladura $f_{C v}$ y el módulo de elasticidad $G_{c}$. mediante las siguientes ecuaciones:

$$
f_{C v}=\frac{F_{u}}{2 \cdot B \cdot e} ;\left(\mathrm{N} / \mathrm{mm}^{2}\right)
$$

Donde:

- $F_{U}$ es la fuerza máxima alcanzada en Newton.

- $A$ es el área de la sección, en milímetros cuadrados $A=B e$ $\left(\right.$ en $\left.\mathrm{mm}^{2}\right)$.

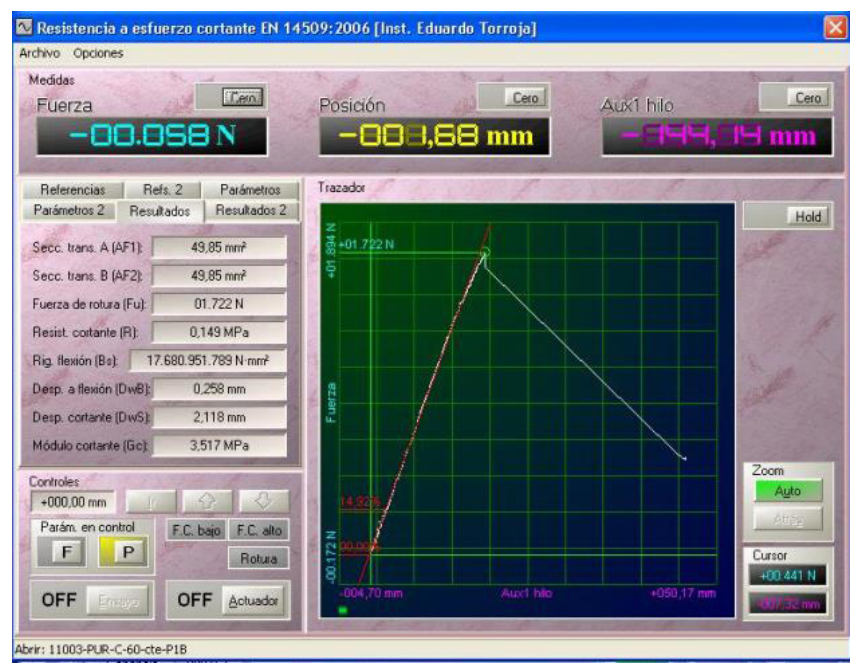

Figura 3. Pantalla de uno de los ensayos a cortante.
- $B$ la anchura en mm.

- $e$ la distancia entre las fibras neutras, centroides de las capas de cobertura en mm (espesor).

- $\Delta w_{s}=$ desplazamiento por esfuerzo cortante para $\Delta F$.

- $d_{C}=d=$ espesor del material del núcleo.

La fórmula para el cálculo del módulo de elasticidad a la cizalladura $G_{C}$ se indica en el apartado A.3.5.2 de la norma:

$$
G_{C}=\frac{\Delta F \cdot L}{6 \cdot B \cdot d_{C} \cdot \Delta \mathrm{w}_{\mathrm{s}}} \quad G_{C}
$$

Donde:

- $\Delta F$ = incremento de la fuerza en la zona elástica considerada (aproximadamente entre el 20 y el $60 \%$ de la fuerza de rotura).

- $L=$ luz entre los apoyos inferiores de los extremos.

- $B=b=$ anchura de la probeta.

\section{RESULTADOS OBTENIDOS}

Hay multitud de ensayos adicionales a los expresados en el apartado anterior que ayudan a definir de una forma más precisa aportando parámetros de indudable importancia. No obstante, en este trabajo no se pretende realizar un informe experimental exhaustivo de todos ellos, pero sí los que pueden afectar más directamente a la resistencia mecánica y que se pueden ver afectados por sus métodos de obtención. En la Figura 3 se muestra la pantalla de la prensa en la que se han realizado los ensayos. Además de los parámetros de los ensayos se muestra una gráfica de carga deformación correspondiente a la probeta 1 . En este caso se detecta la rotura para una carga de $1,722 \mathrm{kN}$. La rotura se alcanza de forma brusca, aunque la probeta mantiene una capacidad de carga remanente debida a las chapas metálicas.

En la Tabla 1 se resumen los resultados obtenidos en los ensayos de los paneles sándwich.

Todas las roturas excepto las de las probetas 2 y 5 del panel se han producido por colapso de la chapa de cobertura superior.

\section{DISCUSIÓN. ANÁLISIS DE LOS RESULTADOS}

$\mathrm{Al}$ realizar el ensayo es necesario que la rotura se produzca por cortante (Figura 4), es decir, que la fuerza ejercida $F_{u}$, combinada con la distancia $L$, entre la fuerza y el apoyo, sea la adecuada para que no se produzca antes el colapso de la chapa superior debido a la tensión de arrugamiento. De la observación de la expresión de $\sigma_{w}$ se deduce que cuanto menor sea la distancia

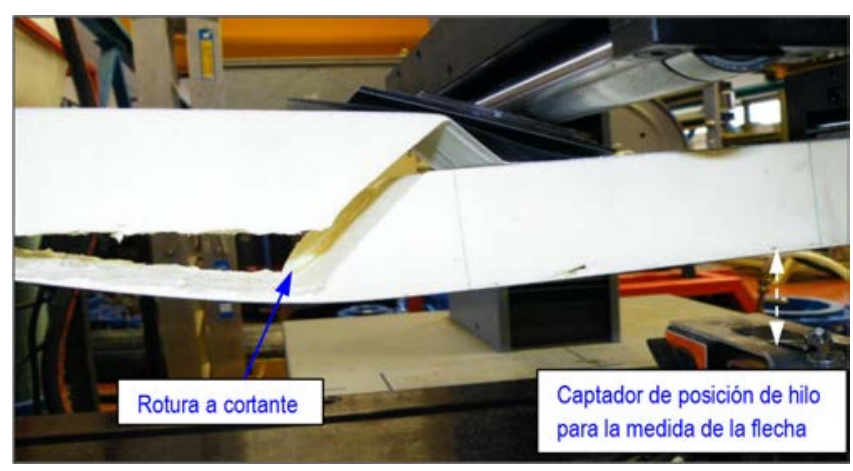

Figura 4. Rotura a cortante, sin colapso de la chapa del panel. 
Tabla 1. Ensayos de cortante sobre probetas de panel CT 60.

\begin{tabular}{|c|c|c|c|c|c|c|}
\hline Probetas & $\begin{array}{c}\text { Resistencia a } \\
\text { esfuerzo } \\
\text { cortante } \\
\mathrm{f}_{\mathrm{Cv}}=\mathrm{k}_{\mathrm{v}} \mathrm{Fu} / 2 \mathrm{Be} \\
(\mathrm{MPa})\end{array}$ & $\begin{array}{c}\text { Logaritmo } \\
\text { neperiano de } \\
f_{\mathrm{Cv}_{\mathrm{v}}} \\
\mathbf{y}=\operatorname{Ln}\left(\mathrm{f}_{\mathrm{Cv}}\right)\end{array}$ & $\begin{array}{l}\text { Módulo de } \\
\text { esfuerzo } \\
\text { cortante } \\
\text { G }_{\mathrm{c}}(\mathrm{MPa})\end{array}$ & $\begin{array}{c}\text { Logaritmo } \\
\text { neperiano de } \\
G_{c} \\
y=\operatorname{Ln}\left(G_{c}\right)\end{array}$ & $\begin{array}{c}\text { Fza } \\
F_{\text {Total }}(\mathrm{N})\end{array}$ & $\begin{array}{c}\text { Longitud } \\
\text { entre } \\
\text { apoyos } \\
L(\mathrm{~mm})\end{array}$ \\
\hline 1 & 0,149 & $-1,904$ & 3,517 & 1,258 & $1.722,0$ & 900 \\
\hline $2^{(*)}$ & 0,165 & $-1,802$ & 3,479 & 1,247 & $1.893,0$ & 900 \\
\hline 3 & 0,164 & $-1,808$ & 3,451 & 1,239 & $1.880,0$ & 900 \\
\hline 4 & 0,169 & $-1,778$ & 3,796 & 1,334 & $1.948,0$ & 800 \\
\hline $5^{(*)}$ & 0,193 & $-1,645$ & 3,545 & 1,266 & $2.270,0$ & 700 \\
\hline 6 & 0,176 & $-1,737$ & 3,381 & 1,218 & $2.041,0$ & 700 \\
\hline 7 & 0,193 & $-1,645$ & 2,985 & 1,094 & $2.221,0$ & 600 \\
\hline Medias & 0,173 & $-1,760$ & 3,451 & 1,236 & $1.996,4$ & \\
\hline$\sigma_{\mathrm{y}}$ : Desviación estandar & 0,016 & 0,093 & 0,243 & 0,073 & 195,4 & \\
\hline \multirow[t]{2}{*}{$x_{p}=(5 \%$ v.f. $)=$ Valor Caracteristico: } & 0,140 & & 2,923 & & & \\
\hline & $\begin{array}{c}\text { Resistencia } \\
\mathrm{f}_{\mathrm{Cv}}(\mathrm{MPa})\end{array}$ & & $\begin{array}{l}\text { Módulo } E \text {. } \\
G_{c}(\mathrm{MPa})\end{array}$ & & & \\
\hline
\end{tabular}

$(*)$ Roturas a cortante.

entre apoyos $L$, menor será la tensión de arrugamiento $\sigma_{w}$ pero también será necesaria una fuerza mayor para provocar la rotura. Estas consideraciones nos indican que para grandes espesores la situación del ensayo se vuelve aún más crítica, ya que también a mayor espesor es necesaria una fuerza mayor.

La tensión de arrugamiento $\sigma_{w}$ se obtiene mediante la determinación del momento flector último $M_{u}$, y a partir de él se calcula $\sigma_{w}$ mediante las siguientes expresiones, en las que:

- L: Longitud entre apoyos.

- $e$ : Espesor de la probeta del panel.

- $A_{1}$ : Área de la sección transversal de la cara a compresión de la chapa superior.

$$
\sigma_{w}=\frac{M_{u}}{e \cdot A_{1}} \quad M_{u}=\frac{F_{u}}{2} \cdot \frac{L}{3}=\frac{F_{u} \cdot L}{6}
$$

$$
\sigma_{w}=\frac{F_{u} \cdot L}{6 \cdot e \cdot A_{1}}
$$

Puesto que los espesores de chapa de los paneles suelen ser los mismos para todos los espesores de panel, el aumento de la fuerza de ensayo contribuye a que se claven los bordes de las placas de apoyo más fácilmente, por lo que se hace imprescindible estudiar los apoyos y ver la forma en la que la fuerza se transmita en toda la superficie de las placas de apoyo y evitar que se canalice de forma puntual a una arista, esto nos proporcionará la posibilidad de que la fuerza de ensayo aumente más y pueda alcanzar la resistencia a esfuerzo cortante del panel que deseamos determinar.

\subsection{Problema con el ensayo de cortante}

El problema que se produce es el siguiente: al aplicar la carga, a medida que aumenta la carga, el panel se curva, pero la placa de reparto de la carga no, por lo que la fuerza que al principio se repartía a lo largo de la longitud de la placa, ahora se concentra de forma estrictamente puntual en los dos bordes de la placa provocando el colapso de la chapa del panel por clavado de la placa.
Cuanto mayor es el espesor de la probeta, mayor es la fuerza necesaria para provocar la rotura a cortante, y a su vez mayor es el riesgo de que la chapa de cobertura del panel colapse, debido a que se supera la tensión de arrugamiento de la misma.

Por lo tanto, un simple ensayo de carga en 4 puntos destinado a realizar una rotura por cortante se ve afectada por una serie de circunstancias imputables al propio ensayo, recordándonos en cierta forma el principio de indeterminación de Heisenberg, en el que el procedimiento de medida afectaba a la propia medida. Esto impide que se pueda determinar la resistencia a cortante real del panel, ya que es necesario ensayar una gran cantidad de probetas hasta conseguir alguna rotura fiable. En la Figura 5 puede observarse cómo se clava literalmente la placa que debía repartir mínimamente la carga y que se convierten en una auténtica carga puntual sobre una arista.

\subsection{Solución que se propone}

Se trata de desarrollar un sistema de ensayo en el que la placa de apoyo y reparto de la carga sea lo suficientemente rígida como para repartir la carga uniformemente, pero lo suficientemente flexible como para acoplarse a la curvatura del panel sin afectar a la misma.

El proceso que se produce actualmente es el siguiente que se muestra en la Figura 6, se comienza a cargar la placa rígida que reparte inicialmente la carga (R1) y a medida que el panel se va curvando la placa no lo hace, por lo que se apoya únicamente en dos aristas sobre las que se aplica toda la carga (R2). A medida que la carga aumenta, la placa se inclina y la carga se desplaza paulatinamente a una de las aristas, hasta concentrarse totalmente sobre ella haciendo colapsar la chapa que envuelve el panel ( $\mathrm{R}_{3}$ ), este efecto de colapso se favorece debido a que el panel está sometido a esfuerzo cortante y momento flector, produciéndose compresión en la cara superior del mismo provocándose el colapso por arrugamiento de la chapa de la cara superior.

Por lo tanto, es necesario desarrollar un sistema de apoyo flexible, como se muestra en la Figura 6, que garantice la distribución de la carga sobre el apoyo (F2) hasta que se alcance un reparto uniforme para que la totalidad de la carga alcance 
la masa del panel y se consiga la rotura del mismo por cortante, objetivo de la medida.

En este punto se propone construir un apoyo de las mismas dimensiones con chapas de pequeño espesor, pero de acero templado, que tengan un elevado límite elástico para que se curve debido al pequeño espesor de cada una de ellas de forma que actúe a modo de ballesta, acoplándose a la curvatura del panel, pero sin punzonarse, permitiendo así el libre giro de la barra cilíndrica sobre la que se aplica la carga. Las chapas han de estar independientes para que permitan el movimiento entre ellas, a lo sumo para evitar deslizamientos incontrolados y poder ubicarlas de forma prismática se

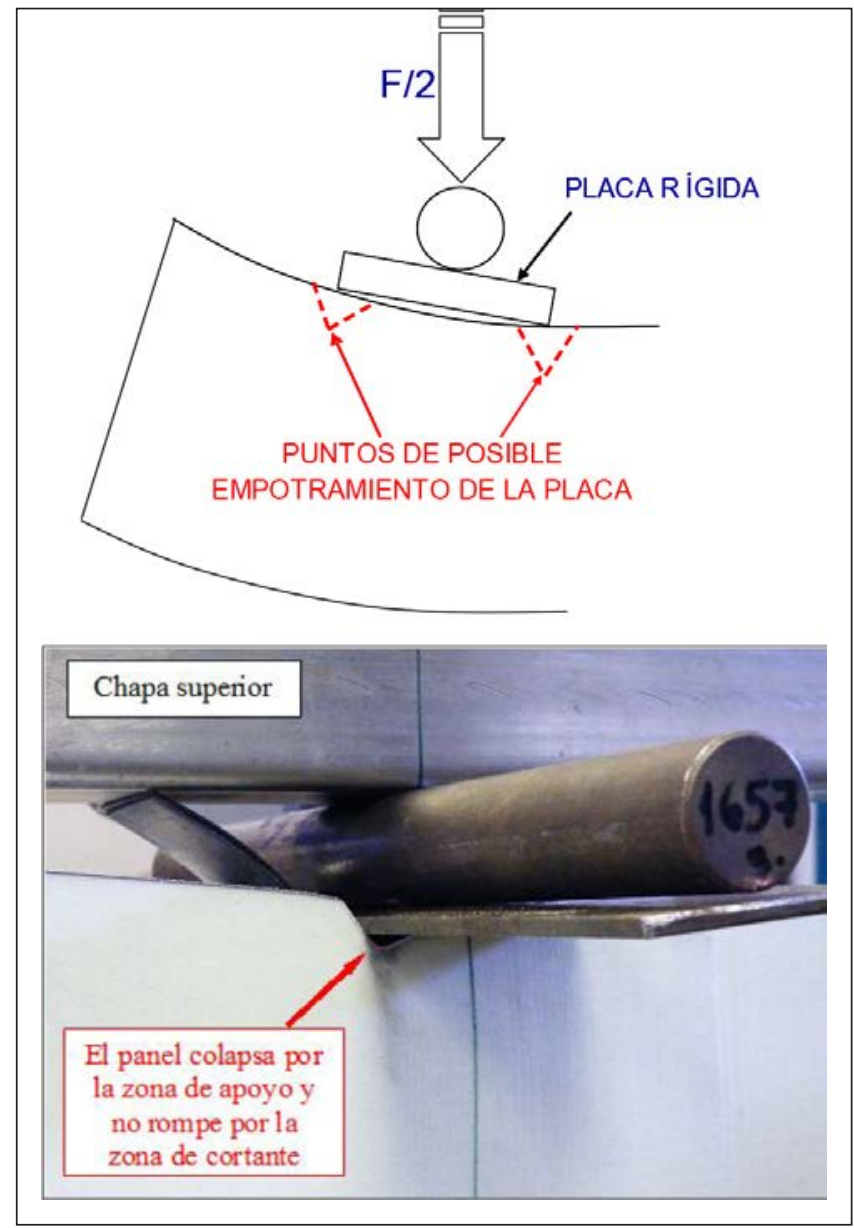

Figura 5. La placa rígida clava sus bordes en la probeta y colapsa la chapa del panel. puede colocar un papel con leve adherencia a doble cara entre ellas que permite mantenerlas unidas, pero que no aporta resistencia mecánica que evite el deslizamiento entre ellas.

En la Figura 7 se observa el efecto que produce la fuerza al aplicarse sobre las chapas de acero.

En la Figura 8 se observa el detalle de una de las soluciones utilizando chapas apiladas en el apoyo de la fuerza para que se adapten al panel cuando se curve por efecto de la misma.

En la Figura 9 se observa que con el nuevo apoyo se consigue que no se produzca el clavado de la placa rígida sobre el panel, tanto en la cara superior como en la inferior.

A partir de aquí es necesario un trabajo de investigación contrastado experimentalmente comparando la tensión de arrugamiento de la chapa del panel con los resultados de la resistencia a esfuerzo cortante del mismo para ver las limitaciones del ensayo en lo referente a los espesores máximos de panel susceptibles de poder ser ensayados.

Una de las posibilidades de estudio es la realización de simulaciones por elementos finitos.

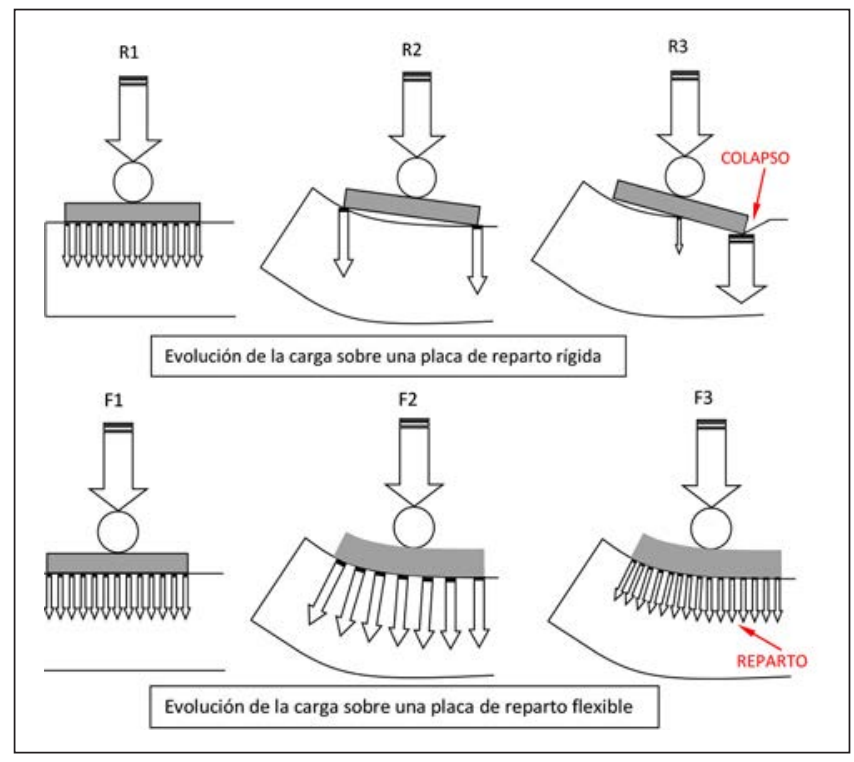

Figura 6. Proceso de clavado de la placa de apoyo. Sistema de apoyo propuesto.

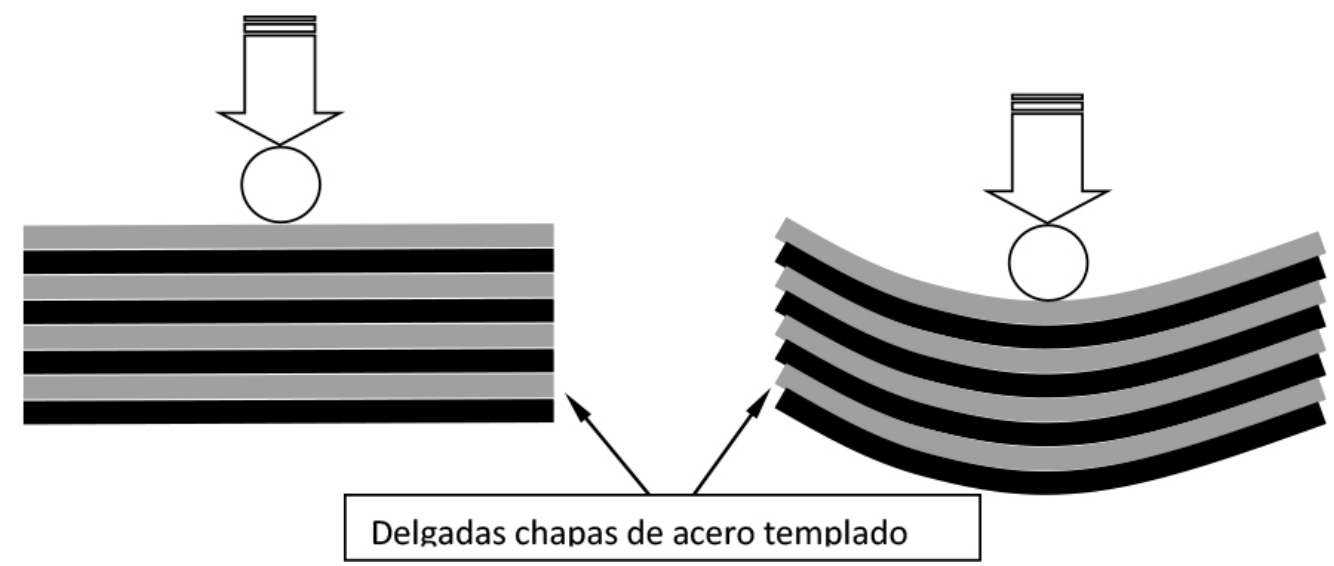

Figura 7. Apoyo con placas flexibles. 


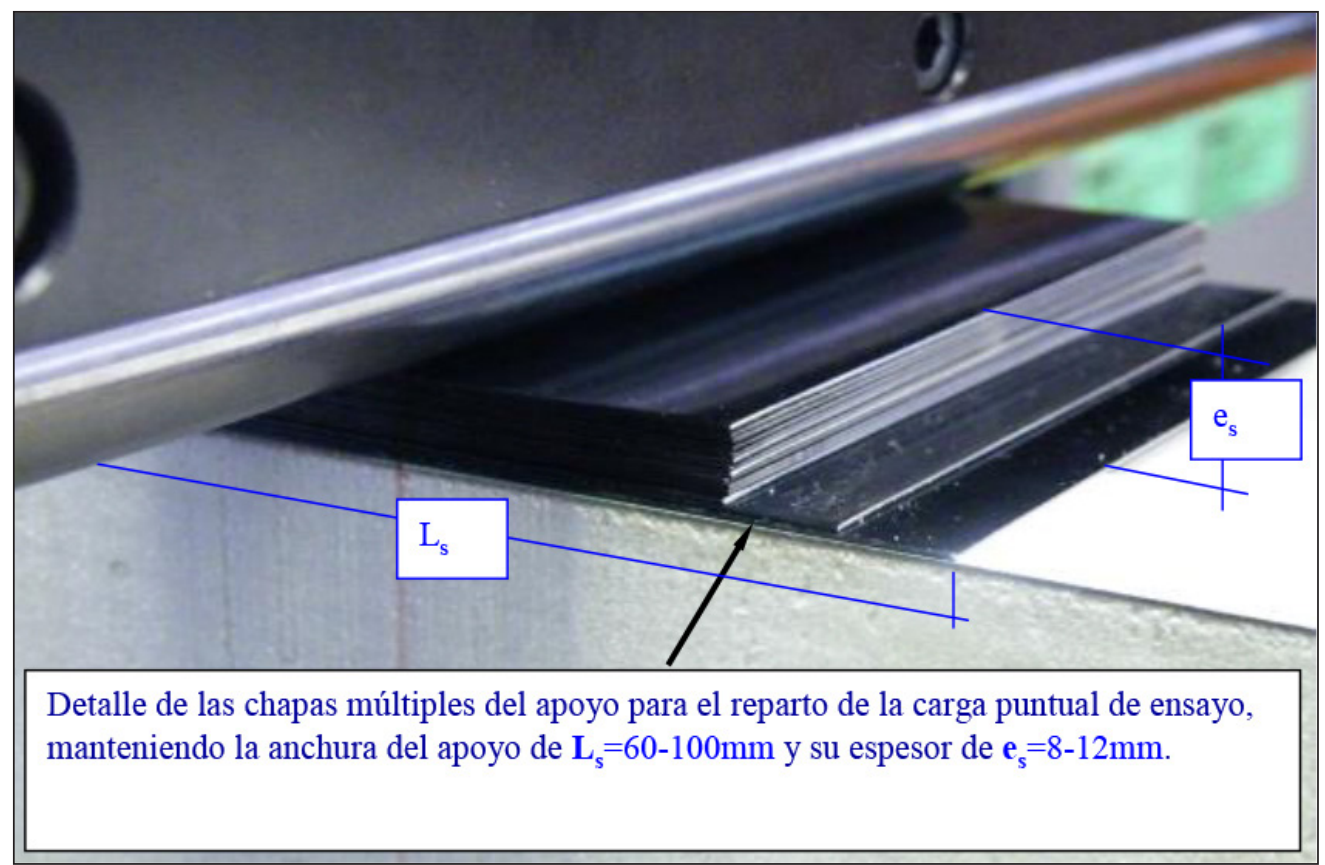

Figura 8. Apoyo con chapas de acero templado.

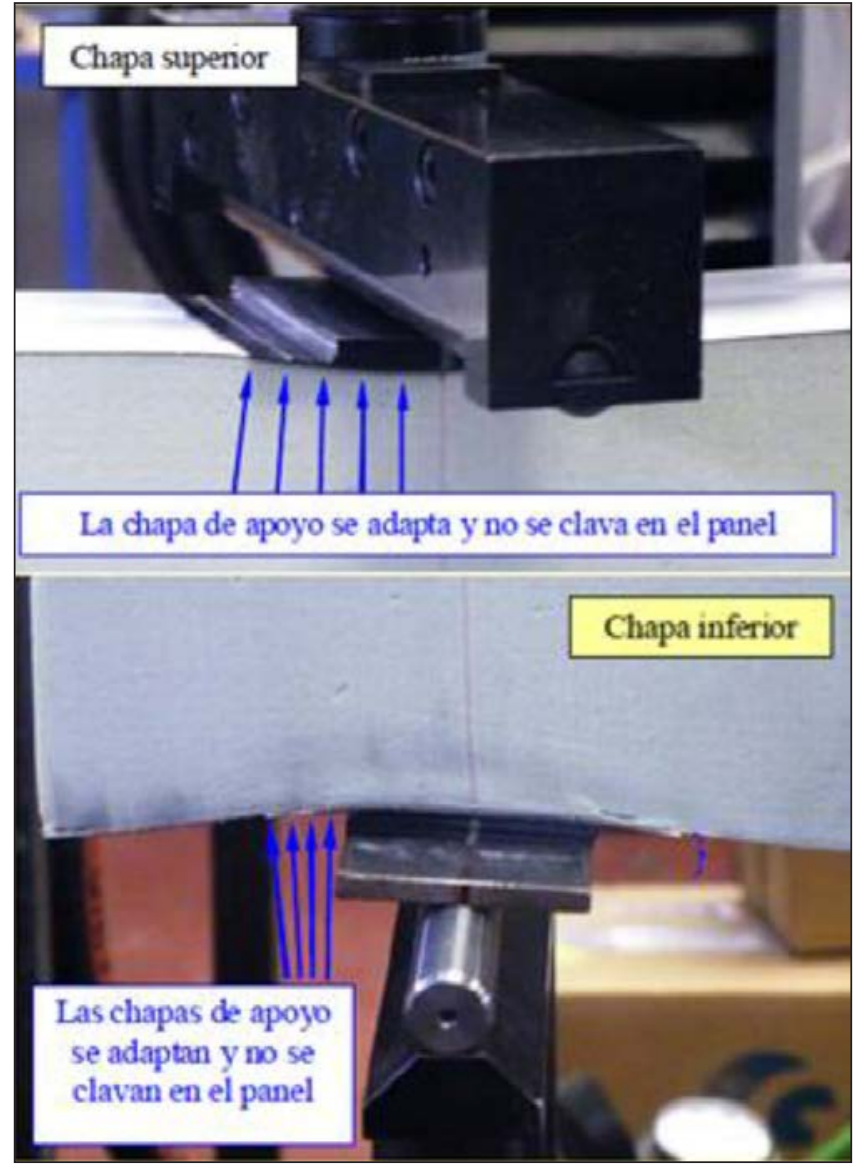

Figura 9. Apoyos superior e inferior con placas flexibles que se adaptan a las superficies.

\subsection{Simulación por elementos finitos}

A la vista de los problemas detectados experimentalmente, y en concreto con los detectados en los apoyos, se ha optado por tratar de simular el ensayo y ver si es posible modelizarlo

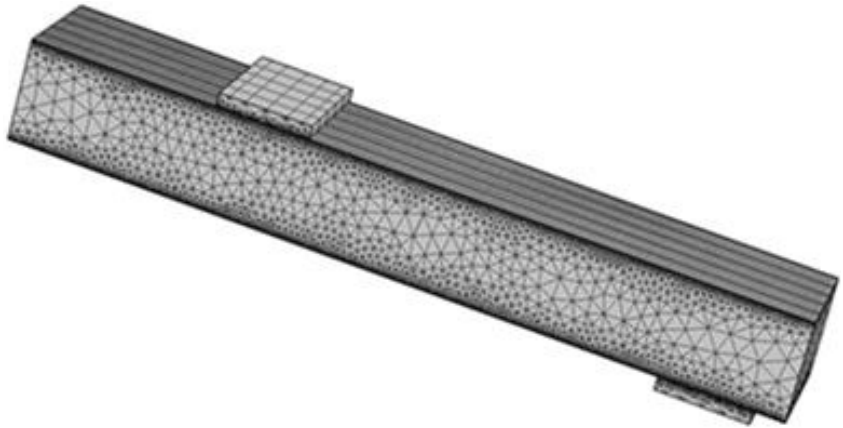

Figura 10. Modelo 3D (media probeta).

evitando así la realización de numerosos ensayos adicionales, realizando tan sólo los de comprobación con el modelo.

Esta simulación se ha realizado con el paquete de software de simulación por elementos finitos COMSOL Multiphysics. Se ha realizado un modelo 3D con un plano de simetría en el centro de la probeta (ver Figura 10). Las capas de cobertura se han simulado como láminas rectas. Se ha considerado un comportamiento elástico lineal de la espuma obtenido en ensayos en el laboratorio y un comportamiento elasto-plástico para las láminas de acero. El modelo ha reproducido las condiciones de ensayo en lo referente a distancias y condiciones de contorno.

Para tener en cuenta el efecto del apoyo se han realizado otros cálculos con chapas múltiples e independientes, hasta completar el mismo espesor de $8 \mathrm{~mm}$, en las siguientes cantidades y espesores: 3 de $0,5 \times 60 \times 100 \mathrm{~mm}$, más 3 de $0,5 \times 55$ $\times 100 \mathrm{~mm}$, más 3 de $0,5 \times 50 \times 100 \mathrm{~mm}$, más 3 de $0,5 \times 45 \times$ $100 \mathrm{~mm}$ y 4 de $0,5 \times 40 \times 100 \mathrm{~mm}$.

Los valores de cargas y deformaciones simulados se comparan con los obtenidos en los experimentos:

$F_{U}=1.700 \mathrm{~N} ; F_{1 / 2}=850 \mathrm{~N} ; \delta=17 \mathrm{~mm} ; L=900 \mathrm{~mm} ; R_{V}=0,15$ $\mathrm{MPa} ; \sigma_{y}=210 \mathrm{MPa}$. 
El movimiento se provoca mediante un desplazamiento del apoyo de la carga hasta conseguir la flecha provocada por la misma, ambos parámetros se han obtenido de los ensayos experimentales.

En la Figura 11 se observa que las aristas de la placa de reparto de la carga superior se produce el clavado de la misma sobre la probeta, superando el límite elástico de la chapa de cobertura del panel (aproximadamente de $210 \mathrm{MPa}$ ) provocando su colapso.

En la Figura 12 se observa que la zona más tensionada de núcleo de espuma de la probeta es la zona de cortante, es decir, entre la carga y el apoyo, en las que se intuyen los posibles caminos de la rotura. Los valores de las tensiones coinciden exactamente con los de la rotura real $R v=0,15 \mathrm{MPa}$.
Esta situación es en la que ya se habría producido dicha rotura, ya que la zona más rojiza de la espuma tiene valores superiores a los 0,15 MPa a los que se produce la rotura real.

En las imágenes de la Figura 13 se observa como en uno de los ensayos realizados se produce el colapso de la chapa del panel. Esta situación ha sido reproducida correctamente en la simulación. En caso de no haberse colapsado la chapa, en este punto se comienza la rotura de la espuma.

En las imágenes de la Figura 14 se muestra un resultado de la simulación análoga a la anterior, sustituyendo una placa gruesa por varias finas.

En estos cálculos se alcanza una carga mayor, lo que permite distinguir las zonas de mayor tensión de la espuma. Se

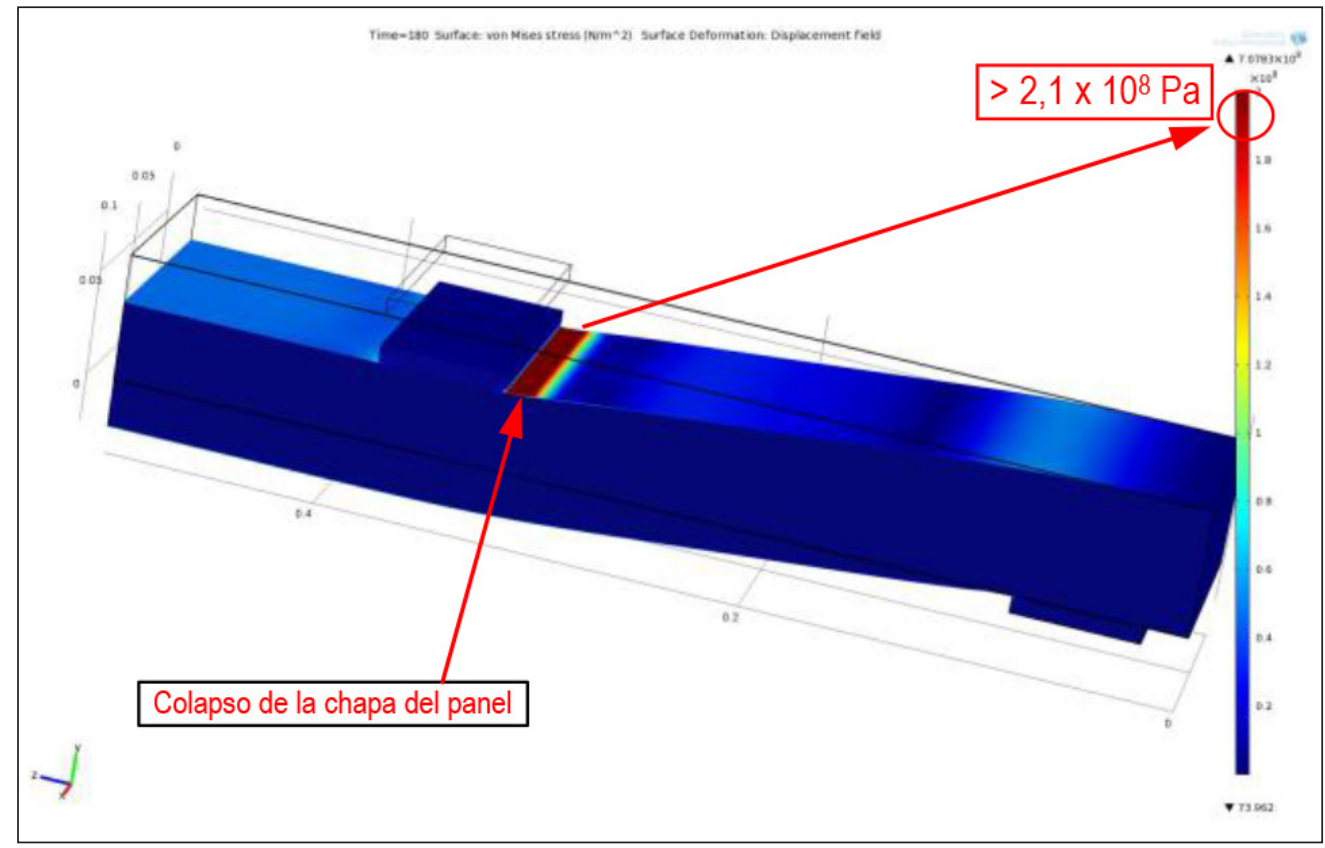

Figura 11. Simulación. Colapso chapa superior del panel con placa de apoyo rígida.

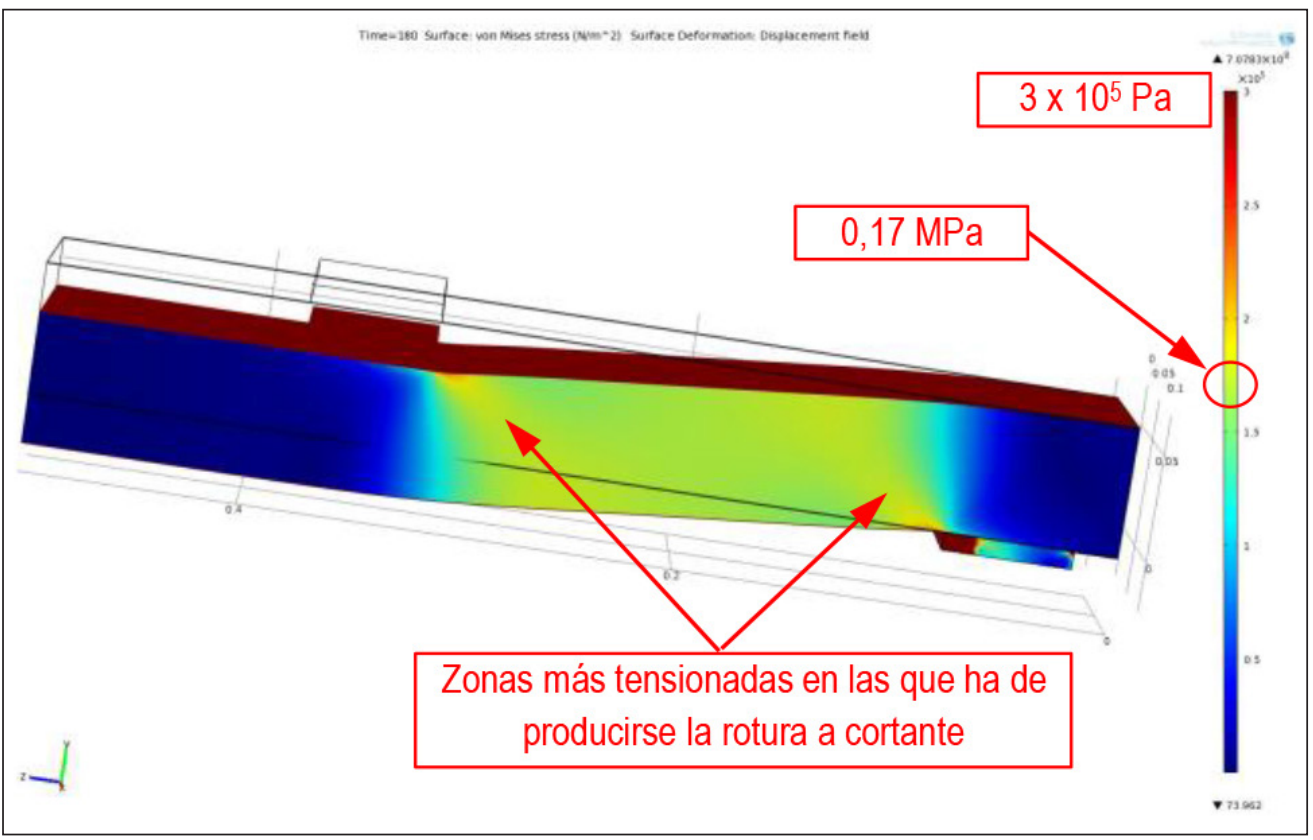

Figura 12. Núcleo sometido a esfuerzo cortante. 


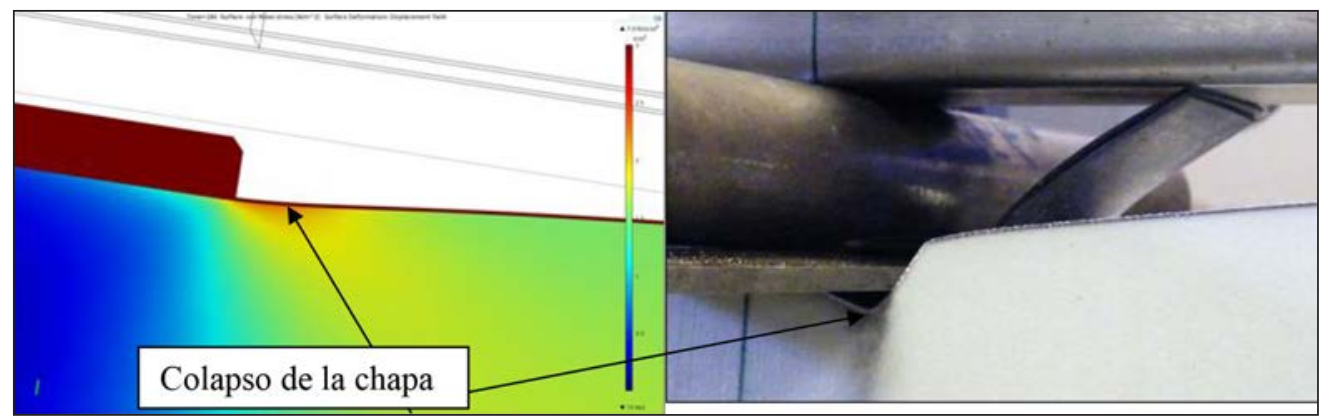

Figura 13. Detalles de la zona de mayor tensión de la espuma aislante por la que se ha de producir la rotura a cortante, pero no se produce por el colapso de la chapa.

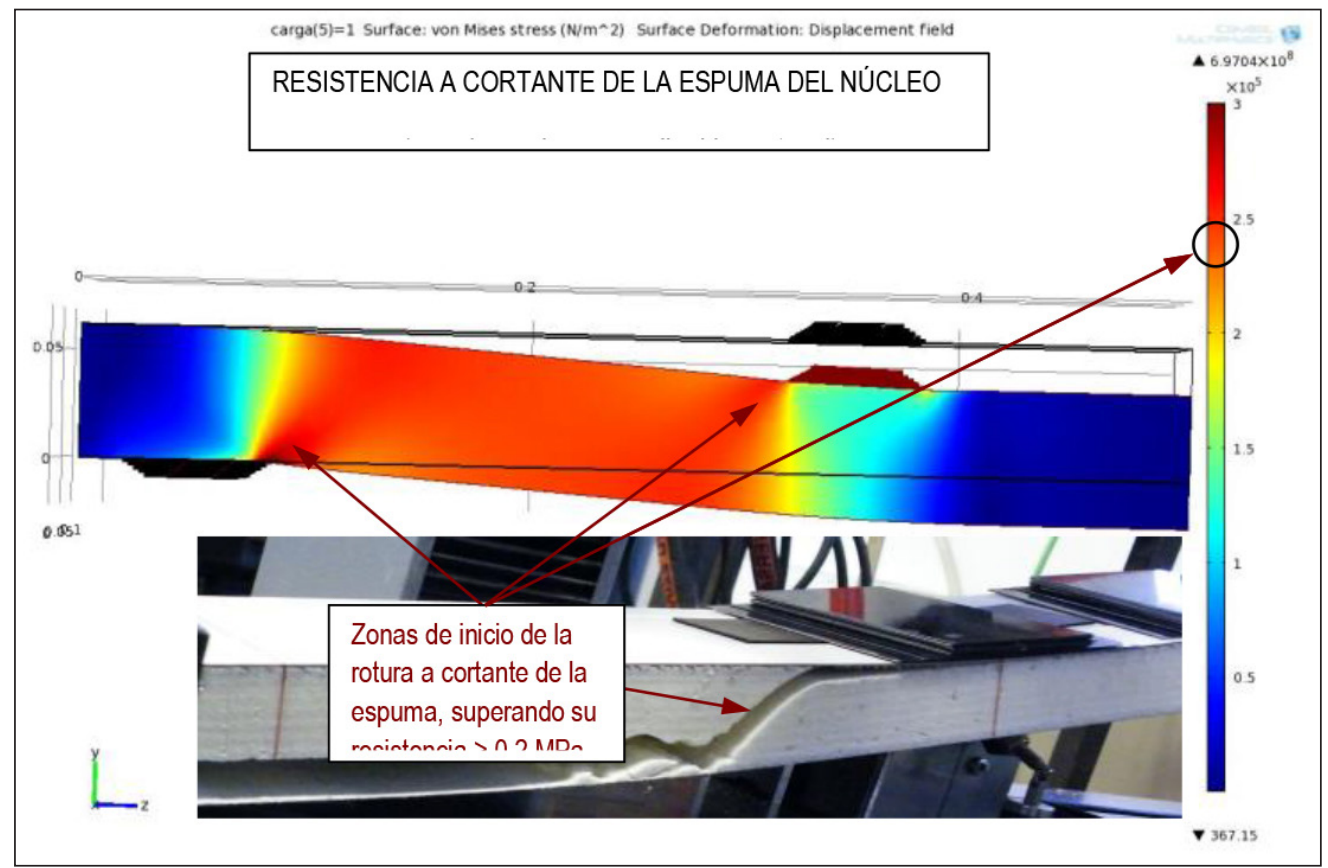

Figura 14. Detalles de la zona de mayor tensión de la espuma aislante por la que se ha de producir la rotura a cortante, y que efectivamente se produce al evitar las chapas flexibles de apoyo el colapso de la chapa.

observa que la tensión de la espuma del panel ha aumentado en la zona de cortante hasta valores próximos a los 0,24 $\mathrm{MPa}$; si recordamos que su rotura real ha sido de o,15 $\mathrm{MPa}$, esta situación está forzada ya que la rotura ya se habría producido.

Todas las distribuciones de tensión en la probeta, tanto en las chapas de cobertura del panel como en la espuma del núcleo, reproducen con bastante aproximación la situación real realizada en los ensayos experimentales, como puede observarse comparando ambas imágenes de la Figura 14.

\section{CONCLUSIONES}

A la vista del estudio realizado y de los resultados obtenidos se puede concluir que:

- La caracterización de este tipo de paneles sándwich compuesto por la unión de varios materiales comporta algunos problemas en la determinación de ciertos parámetros que definen sus características funcionales.

- Los ensayos fijados por las normas de aplicación son adecuados, pero han de observarse ciertos detalles que pueden afectar a los resultados:
- Resistencia a esfuerzo cortante: El tipo de placas de reparto de la carga y el de apoyos ha de optimizarse para evitar el colapso de las capas de cobertura del panel La solución propuesta de sustituir el apoyo rígido de una chapa por otro con varias, lo evita.

En el presente trabajo se han realizado una serie de simulaciones por elementos finitos. Se ha demostrado que estas simulaciones reproducen en gran medida los ensayos experimentales y suponen una gran ayuda en la caracterización, si bien es necesario contrastar las mismas con pruebas reales.

A la vista de los resultados obtenidos se demuestra la necesidad de importantes trabajos de investigación tanto para el desarrollo de modelos más exactos como para la definición de procedimientos de medida más adecuados.

En cuanto a los ensayos experimentales en un futuro próximo se hace necesario el optimizar las condiciones de los ensayos y su análisis.

- Mejorar el sistema de apoyo con chapas múltiples (cantidad, tipo de acero, etc.).

- En las simulaciones se abre un capítulo muy interesante con un gran trabajo por hacer hasta conseguir reproducir 
lo más exactamente posible todos los procesos que ocurren en los ensayos:

- En concreto definir en el modelo las zonas «frontera» de contacto entre los materiales.

- Definir exactamente en el modelo las secciones perfiladas de las capas de cobertura del panel.

- Finalmente y abundando en el campo de la simulación, existen una serie de ensayos de largo plazo, sobre probetas y panel completo, en los que se ha de someter a carga constante a los mismos durante tiempos prolongados, hasta alcanzar deformaciones límite e incluso hasta llegar a rotura, por lo que la simulación de este tipo de pruebas ahorraría mucho tiempo y podrían realizarse cambiando las condiciones de contorno sin necesidad de esperar meses hasta obtener resultados.

- Riesgos técnicos:

El mayor riesgo técnico identificado radica en la dificultad de realizar correctamente la definición del modelo teórico para la adecuada interpretación por software de simulación por elementos finitos y su más exacta correlación con el modelo real.

Para minimizar este riesgo se deberán realizar diferentes modelos teóricos y contrastarlos inicialmente con ensayos reales.

\section{REFERENCIAS}

(1) ECCS (European Convention for Constructional Steelwork). Preliminary European Recommendations for Sandwich Panels. T.C.7-W.G 7.4.

(2) Directiva 89/106/CEE del Consejo de 21 de diciembre de 1988 relativa a la aproximación de las disposiciones legales, reglamentarias y administrativas de los Estados miembros sobre los productos de construcción.

(3) EOTA (European Organisation for Technical Approvals). Guía n. ${ }^{0} 16$.

(4) UNE 41950:1994 Panel de poliuretano con capas de cobertura metálicas o no metálicas. CTN: AEN/CTN 41 - CONSTRUCCIÓN.

(5) UNE-EN 14509:2014 Paneles sandwich aislantes autoportantes de doble cara metálica. Productos hechos en fábrica. Especificaciones. CTN: AEN/CTN 41/SC 4 - CUBIERTAS.

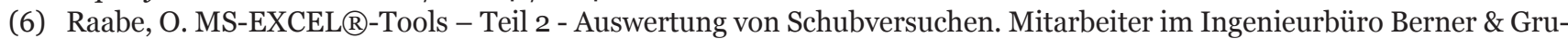
ber GmbH, Elisabethenstr. 62, 64283 Darmstadt und freier Mitarbeiter im iS-mainz.

(7) Martikainen, L., Hassinen, P. (1996). Load-bearing capacity of continuous sandwich panels. Report 135. Helsinki University of Technology, Department of Structural Engineering.

(8) Berner, K. (1994). Erarbeitung vollständiger Bemessungsgrundlagen im Rahmen bautechnischer Zulassungen für Sandwichbauteile. Teil 2: Berücksichtigung zusätzlicher Beanspruchungen bei der Bemessung von durchlaufenden Sandwichplatten im Zwischenstützbereich. Mainz: FH des Landes Rheinland-Pfalz, KartoniertFraunhofer IRB Verlag.

(9) Allen, G. (1969). Analysis and Design of Structural Sandwich Panel. Oxford, UK: Pergamon Press.

(10) Zenkert, D. (1995). An Introduction to Sandwich Construction. Solihull, UK: EMAS.

(11) Grédiac, M. (1993). A finite element study of the transverse shear in honeycomb cores. International Journal of Solids and Structures, 30(13): 1777-1788.

(12) Shi, G., Tong, P. (1995). Equivalent transverse shear stiffness of honeycomb cores. International Journal of Solids and Structures, 32(10): 1383-1393.

(13) Becker, W. (1998). The in-plane stiffness of honeycomb core including the thickness effect. Archives of Applied Mechanics, 68: 334-341.

(14) Xu, F. X., Qiao, P. (2002). Homogenized elastic properties of honeycomb sandwich with skin effect. International Journal of Solids and Structures, 39: 2153-2188.

(15) Meraghni, F., Desrumaux, F., Benzeggagh, M. L. (1999). Mechanical behaviour of cellular core for structural sandwich panels. Composites: Part A, 30: 767-779.

(16) Balawi, S., Abot, J. L. (2008). The effect of honeycomb relative density on its effective in-plane elastic moduli: an experimental study. Composite Structures, 84(4): 293-299.

(17) Noor, A. K., et al. (1990). Assessment of computational models for multilayered anisotropic plates. Composite Structures, 14: 233-265.

(18) Reddy, J. N. (1984). A simple higher-order theory of laminated composite plate. Journal of Applied Mechanics, 51: 745752.

(19) EuroComposites, S. A., ECA. Honeycomb Data Sheet.

(20) Gibson, L. J., Ashby, M. F. (1997). Cellular Solids: Structure and Properties (2. ${ }^{a}$ edición). Cambridge, UK: Cambridge University Press.

(21) Masters, I. G., Evans, K. E. (1996). Models for the elastic deformation of honeycombs. Composite Structures, 35: 403442.

CTE Código Técnico de la Edificación.

Ensayos realizados en el Laboratorio de Instalaciones del Instituto Eduardo Torroja del CSIC. 